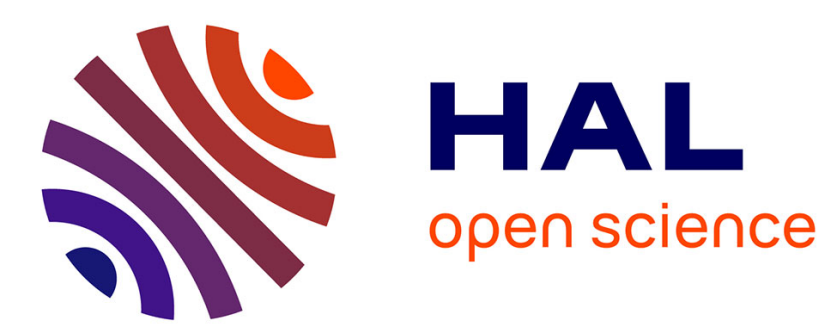

\title{
Nature cristallo-physico-chimique de l'interface entre un semiconducteur et son oxyde propre. I. - Etudes expérimentales des semiconducteurs des groupes A4 et A3 B5 \\ O.V. Romanov
}

\section{To cite this version:}

O.V. Romanov. Nature cristallo-physico-chimique de l'interface entre un semiconducteur et son oxyde propre. I. - Etudes expérimentales des semiconducteurs des groupes A4 et A3 B5. Revue de Physique Appliquée, 1984, 19 (5), pp.379-388. 10.1051/rphysap:01984001905037900 . jpa-00245206

\section{HAL Id: jpa-00245206 https://hal.science/jpa-00245206}

Submitted on 1 Jan 1984

HAL is a multi-disciplinary open access archive for the deposit and dissemination of scientific research documents, whether they are published or not. The documents may come from teaching and research institutions in France or abroad, or from public or private research centers.
L'archive ouverte pluridisciplinaire HAL, est destinée au dépôt et à la diffusion de documents scientifiques de niveau recherche, publiés ou non, émanant des établissements d'enseignement et de recherche français ou étrangers, des laboratoires publics ou privés. 
Classification

Physics Abstracts

$73.20-73.30-73.40 \mathrm{M}-81.60$

\title{
Nature cristallo-physico-chimique de l'interface entre un semiconducteur et son oxyde propre.
}

\section{I. - Etudes expérimentales des semiconducteurs des groupes $\mathbf{A}^{4}$ et $\mathbf{A}^{3} \mathbf{B}^{5}$ (*)}

\author{
O. V. Romanov \\ Laboratoire d'Electronique des Corps Solides, Institut de Physique, Université d'Etat de Leningrad, \\ 199164 Leningrad, URSS
}

(Reçu le 3 juin 1983, révisé le 21 octobre, accepté le 27 octobre 1983)

\begin{abstract}
Résumé. - On présente les résultats d'études systématiques des propriétés électrophysiques et physicochimiques de l'interface oxyde propre anodique ultramince ( 0 à $50 \AA$ )-semiconducteur (OPS), obtenus en étudiant les effets de champ électrique $\left(C, \Delta C, \sigma_{\square}, \Delta \sigma_{\square}, j, \Delta j \sim f(\phi, t)\right)$ dans différents électrolytes aqueux pour un ensemble de semiconducteurs bien choisis des groupes $\mathrm{A}^{4}(\mathrm{Si}, \mathrm{Ge})$ et $\mathrm{A}^{3} \mathrm{~B}^{5}(\mathrm{InP}, \mathrm{InAs}$. InSb; $\mathrm{GaN}, \mathrm{GaP}, \mathrm{GaAs}, \mathrm{GaSb})$. On a utilisé les surfaces (100) et (111) pour le groupe $A^{4}$ et les surfaces (111) et $(\overline{111})$ pour le groupe $A^{3} B^{5}$. Les résultats des expériences indiquent la formation d'une couche électrique spécifique à la première étape de formation de l'interface OPS, dont la grandeur algébrique dépend des propriétés cristallographiques, physiques et chimiques de la surface étudiée. Dans des conditions identiques, sur le système électrolyte-oxyde propre-semiconducteur, on a réussi à mettre en évidence les règles de variation de la double couche électrique à l'interface OPS pour les semiconducteurs des groupes $\mathrm{A}^{4}$, $\mathrm{InB}^{5}$ et $\mathrm{GaB}^{5}$ pour diverses surfaces cristallographiques et en étudiant aussi l'adsorption de métaux lourds $(\mathrm{Cu}, \mathrm{Ag}, \mathrm{Au}, \mathrm{Fe}, \mathrm{Co}, \mathrm{Ni}, \mathrm{Pt}$, etc.). Quelques-unes de ces règles s'observent aussi systématiquement pour l'interface oxyde propre thermique-silicium, ce qui souligne la nature générale de l'interface OPS formée par oxydation du semiconducteur. Dans cette première partie nous présentons l'ensemble des principaux résultats expérimentaux (dont certains sont inédits), relatifs à l'interface OPS pour les semiconducteurs des groupes $\mathrm{A}^{4}$ et $\mathrm{A}^{3} \mathrm{~B}^{5}$, dont l'interprétation générale sera donnée dans la deuxième partie.
\end{abstract}

\begin{abstract}
The processes of the electrochemical oxidation for different semiconductors are investigated in the details, owing to a new experimental approach of the problems of the depth of localization, the origin of a builtin electric charge in an oxide formed from a semiconductor at an insulator-semiconductor interface, and the origin of surface states at the same interface. Field effect experiments $\left(C, \Delta C, \sigma_{\square}, \Delta \sigma_{\square}, j, \Delta j \sim f(\phi, t)\right)$ were carried out with semiconductors selected from groups $A^{4}\left(S i, G e\right.$ with surfaces (100) and (111)) and $A^{3} B^{5}(\operatorname{InP}, \operatorname{InAs}, \operatorname{InSb}$; $\mathrm{GaN}, \mathrm{GaP}, \mathrm{GaPs}, \mathrm{GaSb}$ ) with surfaces (111)- $\mathrm{A}^{3}$ and $(\overline{111})-\mathrm{B}^{5}$ in several aqueous electrolytes, pure or contaminated by different metals $(\mathrm{Cu}, \mathrm{Ag}, \mathrm{Au}, \mathrm{Pt}, \mathrm{Pd}, \mathrm{Fe}, \mathrm{Co}, \mathrm{Ni}$, etc...). The results allow to establish the main rules governing the systematic variations of a double electrical layer at the interface with regard to structural, physical and chemical properties of the semiconductor and its own oxide.
\end{abstract}

\section{Introduction.}

Il est impossible de sous-estimer l'importance de l'interface isolant-semiconducteur dans les recherches fondamentales sur les semiconducteurs, aussi bien que dans les applications techniques des systèmes

(*) Cet article a été rédigé alors que l'auteur était en visite au Laboratoire de Physique des Composants à Semiconducteurs (ERA CNRS No 659) de l'ENSERG, Institut National Polytechnique de Grenoble. métal-isolant-semiconducteur (MIS) en microélectronique moderne $[1,2]$. On a déjà utilisé et proposé diverses méthodes expérimentales et considérations théoriques pour les études des systèmes isolantsemiconducteur et MIS [1, 4]. On doit cependant constater l'absence d'une conception ou d'un modèle global de l'interface isolant-semiconducteur utilisable non seulement pour expliquer les résultats expérimentaux, mais aussi pour prévoir les conditions de préparation d'interfaces de meilleure qualité du point de vue de leurs propriétés électrophysiques. Il nous 
semble que la cause principale de cette situation est la concentration des recherches technologiques et même fondamentales sur le Si (matériau privilégié de la microélectronique moderne), alors qu'il serait préférable d'effectuer des études dans des conditions identiques, en utilisant une même méthode, pour différents semiconducteurs convenablement choisis.

Nous présentons et analysons des résultats expérimentaux originaux relatifs aux propriétés électrophysiques et physico-chimiques de l'interface entre un semiconducteur et son oxyde propre anodique obtenus dans des conditions identiques avec des matériaux des groupes $A^{4}(S i, G e)$ et $A^{3} B^{5}(I n P$, InAs, $\mathrm{InSb}$; GaN, GaP, GaAs, GaSb), qui correspondent à des configurations analogues dans le tableau de Mendeleev et dont les propriétés cristallo-physicochimiques varient régulièrement dans chaque groupe. Il est important de souligner que le système oxyde propre-semiconducteur (OPS) est le plus important des interfaces isolant-semiconducteurs car il est souvent utilisé dans la préparation des circuits intégrés, et qu'il est toujours réalisé sur la surface des divers semiconducteurs après leurs traitements physicochimiques. Le but principal de notre travail est de présenter une première approche d'une conception générale de l'interface OPS formée lors du processus d'oxydation (électrochimique ou thermique) de différents semiconducteurs, sur la base des résultats expérimentaux systématiques et originaux relatifs à quelques groupes de matériaux semiconducteurs.

La possibilité d'une telle approche nouvelle se base sur trois remarques. Premièrement, il est maintenant possible de réaliser des recherches systématiques dans ce domaine en utilisant un grand nombre de semiconducteurs monocristallins avec différentes orientations cristallographiques de leurs surfaces.

Deuxièmement, on peut désormais utiliser une méthode effective d'étude commune (in situ) pour ces différents semiconducteurs lors de la formation sur leur surface des couches minces d'oxyde propre anodique, à savoir l'effet complexe de champ électrique en milieu électrolytique $[5,6]$. Cette méthode permet de mesurer simultanément et en permanence les caractéristiques électrophysiques et physicochimiques du système électrolyte-oxyde propre anodique-semiconducteur (EOPS) ou des systèmes électrolytesemiconducteur : tension-capacité $C(\phi)$, tensionphoto-capacité $\Delta C(\phi)$, tension--onductivité longitudinale du semiconducteur $\sigma_{\square}(\phi)$, tension-photoconductivité $\Delta \sigma_{\square}(\phi)$, tension-courant à travers l'interface $j(\phi)$, tension-photocourant $\Delta j(\phi)$, tension-effet de magnétorésistance transversale (ou longitudinale) $\Delta R_{\mathrm{H}} / R_{0}(\phi)$ [5-7]. Les résultats expérimentaux nous permettent alors de discuter les principaux processus électrophysiques (capture, recombinaison et diffusion des porteurs libres de charge électrique) et physicochimiques (transformation du réseau cristallin $\mathrm{du}$ semiconducteur en oxyde propre anodique ou réduc- tion de l'oxyde par un processus électrochimique cathodique).

Troisièmement, il existe maintenant beaucoup de résultats d'analyses électrophysiques et physicochimiques du système $\mathrm{Si}_{-} \mathrm{SiO}_{2}$ (thermique) utilisant des méthodes modernes sous vide : spectroscopie de masse d'ions secondaires (SIMS), spectroscopie Auger, spectroscopie électronique, photoélectronique et de rayons $X$ pour l'analyse chimique (ESCA), etc. $[3,4,8]$.

Lors de nos recherches nous avons utilisé les semiconducteurs suivants : $\mathrm{n}-\mathrm{Si}, \mathrm{p}-\mathrm{Si}, \mathrm{n}-\mathrm{Ge}$ et $\mathrm{p}-\mathrm{Ge}$ de différentes résistivités, et deux orientations de leur surface, selon les plans (111) et (100) : n-InP $\left(N_{\mathrm{D}}=4,6 \times 10^{16} \mathrm{~cm}^{-3}\right) ; \mathrm{n}$-InAs $\left(N_{\mathrm{D}}=2 \times 10^{16}\right.$ $\left.\mathrm{cm}^{-3}\right) ; \mathrm{p}-\operatorname{InSb}\left(N_{\mathrm{A}}=2 \times 10^{12} \mathrm{~cm}^{-3}\right) ; \mathrm{n}-\operatorname{InSb}\left(N_{\mathrm{D}}=\right.$ $\left.1 \times 10^{14} \mathrm{~cm}^{-3}\right) ; \mathrm{n}-\mathrm{GaN}\left(N_{\mathrm{D}} \sim 10^{18} \mathrm{~cm}^{-3}\right) ; \mathrm{n}-\mathrm{GaP}$ $\left(N_{\mathrm{D}}=10^{17} \mathrm{~cm}^{-3}\right) ; \mathrm{n}$-GaAs $\left(N_{\mathrm{D}}=10^{17} \mathrm{~cm}^{-3}\right)$ et $\mathrm{n}$-GaSb $\left(N_{\mathrm{D}} \simeq 10^{19} \mathrm{~cm}^{-3}\right)$, dont les surfaces étudiées étaient orientées selon les plans (111)- $\mathrm{A}^{3}$ et $(\overline{111}) B^{5}\left({ }^{1}\right)$.

On a utilisé des électrolytes aqueux $0,1 \mathrm{~N} \mathrm{H}_{2} \mathrm{SO}_{4}$; $0,1 \mathrm{~N} \mathrm{Na}_{2} \mathrm{SO}_{4} ; 0,1 \mathrm{~N} \mathrm{NaOH}$ en général, et en plus $\mathrm{HF}, \mathrm{HBF}_{4}, \mathrm{NH}_{4} \mathrm{OH}$, etc. dans certains cas particuliers. Le potentiel électrochimique de l'électrode semiconductrice est mesuré habituellement par rapport à une électrode de référence de chlorure d'argent, sauf pour des solutions $\mathrm{HF}$, où il a été mesuré par rapport au platine.

\section{Résultats sur les semiconducteurs élémentaires $\mathrm{A}^{4}$ (Si, Ge).}

Le silicium et le germanium représentent deux exemples différents et importants du compórtement des semiconducteurs dans le système EOPS (Figs. 1 à 8).

Il est possible d'obtenir dans une solution de $\mathrm{HF}$ une surface de Si totalement libre d'oxyde propre. Dans ces conditions, les caractéristiques $C(\phi)$ et $j(\phi)$ (Figs. 1 et 2) sont réversibles et sans hystérésis, ce qui montre la stabilité de la structure chimique de la surface, correspondant plus probablement à des liaisons Si-F. Les dépendances expérimentales $C(\phi)$ et $\sigma_{\square}(\phi)$ (Figs. 1 et 3) coïncident bien avec les dépendances théoriques $C_{\mathrm{sc}}\left(V_{\mathrm{s}}\right)$ et $\sigma_{\square}\left(V_{\mathrm{s}}\right)$, trouvées par résolution de l'équation de Poisson dans des conditions de désertion en négligeant totalement les porteurs minoritaires par suite du non-équilibre thermodynamique. Ce résultat, de même que la bonne correspondance des potentiels de surface $\left(V_{s}\right)$ déterminés d'après les dépendances expérimentales $C(\phi)$ et $\sigma_{\square}(\phi)$ dans une échelle assez vaste de variation du potentiel électrochimique $(\phi)$ du Si (Fig. 3) prouvent que dans ces conditions la tension appliquée est totalement

( $\left.{ }^{1}\right)$ La surface de GaN est orientée selon le plan (21̄01). 


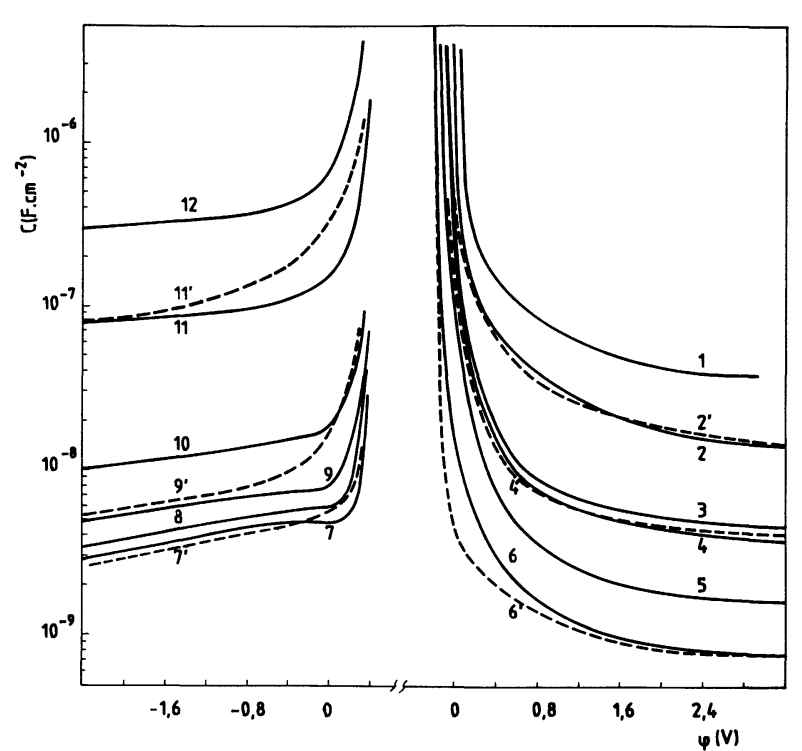

Fig. 1. - Dépendances $C(\phi)$ pour le système « surface (111) de Si/HF » : $1:$ n-Si (0,2 $\Omega$-cm); 2 : n-Si (1); $3:$ n-Si (5); 4 : n-Si (10); 5 : n-Si (45); 6 : n-Si (200); 7 :p-Si (40); 8 : p-Si (20); 9 : p-Si (10); $10:$ p-Si (2); 11 : p-Si $(0,2): 12$ : $\mathrm{p}$-Si $(0,02) ; 2^{\prime}, 4^{\prime}, 6^{\prime}, 7^{\prime}, 9^{\prime}, 11^{\prime}:$ dépendances théoriques correspondantes.

$[C(\phi)$ dependence for the « (111) Si surface/HF » system; $2^{\prime}, 4^{\prime}, 6^{\prime}, 7^{\prime}, 9^{\prime}, 11^{\prime}$ : corresponding theoretical dependences.]

localisée sur la zone de charge d'espace à la surface du Si $[6,7]$

$$
\Delta \phi=V_{\mathrm{s}} .
$$

Alors les fonctions $C(\phi), \sigma_{\square}(\phi)$ et $j(\phi)$ (Fig. 1 à 3) nous montrent que le système $\mathrm{Si} / \mathrm{HF}$ est en fait une jonction de Schottky en milieu liquide, pour laquelle la dépendance $C^{-2} \propto \phi$ est bien vérifiée pour les échantillons de silicium de dopages différents dans la gamme $10^{13} \leqslant N_{\mathrm{D}}, N_{\mathrm{A}} \leqslant 10^{19} \mathrm{~cm}^{-3}$ (Fig. 4). Le sens direct de cette jonction correspond à une polarisation cathodique pour du $\mathrm{n}-\mathrm{Si}$ et à une polarisation anodique pour du $\mathrm{p}-\mathrm{Si}$, et on a alors une couche d'accumulation sur la surface du Si pour chaque type de conductivité $\left(j_{\text {dir }}>10^{-3} \mathrm{~A} . \mathrm{cm}^{-2}\right)$. Le sens inverse correspond à une polarisation inverse pour les deux types de silicium, et on obtient à la surface du Si une couche de désertion en déséquilibre thermodynamique $\left(j_{\text {inv }}<1 \times 10^{-6} \mathrm{~A} . \mathrm{cm}^{-2}\right)$. En ce cas l'intensité du flux électrique $(j)$ est déterminée par le transport de porteurs minoritaires de charge électrique et nous avons observé un photocourant, dont la valeur était proportionnelle à l'intensité d'éclairement (Fig. 2).

L'absence d'oxyde propre sur la surface du Si dans ce cas peut être confirmée par les mesures de la capacité du système [9] :

$$
d_{\mathrm{ox}}<\frac{\varepsilon_{0} \varepsilon_{\mathrm{ox}}}{\max C}=3 \text { à } 5 \AA,
$$

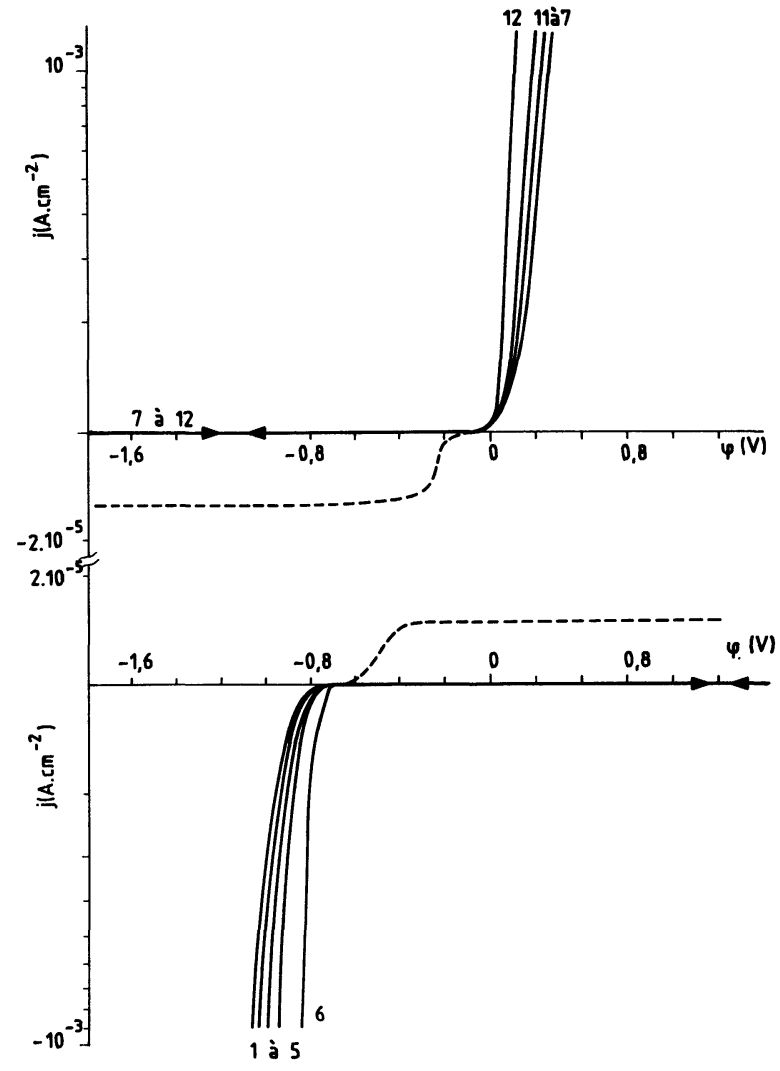

Fig. 2. - Dépendances $j(\phi)$ pour le système « surface (111) de $\mathrm{Si} / \mathrm{HF}$ ». Les notations sont les mêmes que sur la figure 1. Le photocourant est indiqué en pointillés.

$[j(\phi)$ dependence for the " (111) Si surface/HF " system; same notations as in figure 1 . The photocurrent is given by the dotted curve.]

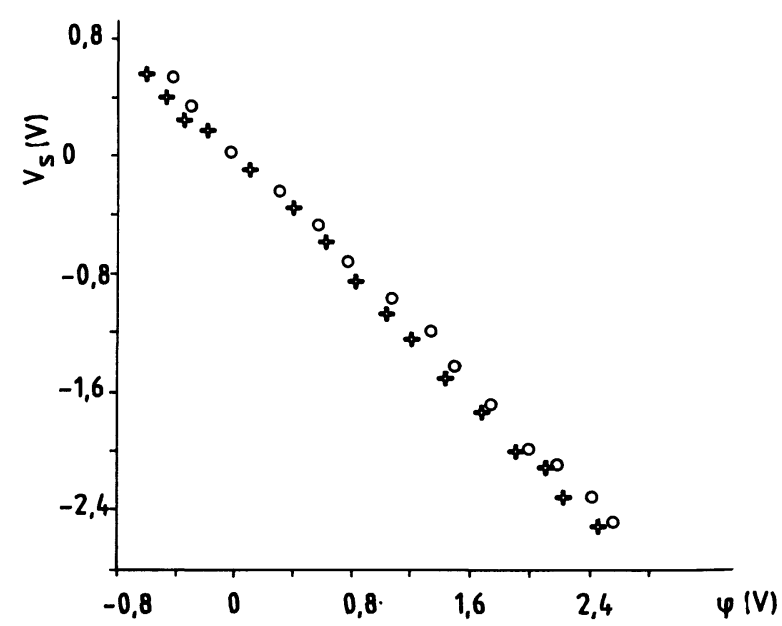

Fig. 3. - Correspondance des variations du potentiel de surface $\left(V_{\mathrm{s}}\right)$ et du potentiel électrochimique $(\phi)$ déduites des mesures de capacité $(O)$ et de conductivité $(x)$ sur l'électrode $\mathrm{n}-\mathrm{Si}(200 \Omega \mathrm{cm})$, surface (111), dans HF.

[Correspondence between surface potential $\left(V_{\mathrm{s}}\right)$ and electrochemical potential $(\phi)$ deduced from capacitance $(O)$ or conductivity $(\times)$ measurements on $\mathrm{n}-\mathrm{Si}(200 \Omega \mathrm{cm})$, (111) surface, in HF.] 


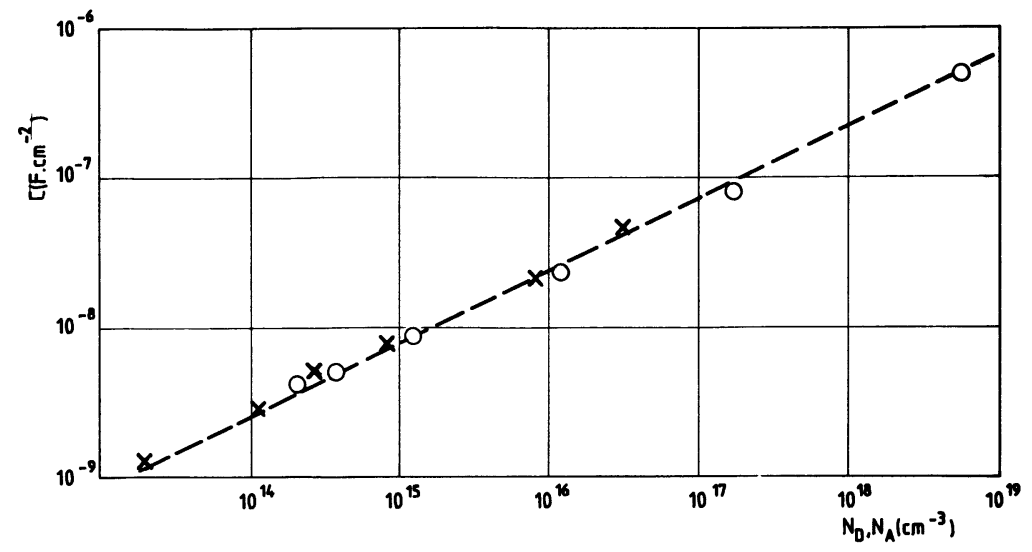

Fig. 4. - Variation de la capacité du système $\mathrm{Si} / \mathrm{HF}$ mesurée pour un potentiel de surface $V_{\mathrm{s}}=1,6 \mathrm{~V}$ avec divers dopages de type $\mathrm{n}(\times)$ ou $\mathrm{p}(\bullet)$. La dépendance théorique de Schottky $\left(N_{\mathrm{A}, \mathrm{D}}=\frac{2\left|V_{\mathrm{s}}\right| C_{\mathrm{sc}}^{2}}{\varepsilon_{0} \varepsilon_{\mathrm{sc}} q}\right)$ est présentée en pointillés.

[Si/HF system capacitance (measured for a surface potential $V_{\mathrm{s}}=1.6 \mathrm{~V}$ ) as a function of $\mathrm{n}(\times)$ or $\mathrm{p}(\bullet)$-type dopings. The dotted line gives the theoretical Schottky dependence : $\left.N_{\mathrm{A}, \mathrm{D}}=2\left|V_{\mathrm{s}}\right| C_{\mathrm{sc}}^{2} / \varepsilon_{0} \varepsilon_{\mathrm{sc}} q.\right]$

avec $d_{\mathrm{ox}}$ : l'épaisseur d'oxyde propre possible; $\varepsilon_{0}:$ permittivité du vide; $\varepsilon_{\mathrm{ox}}$ : constante diélectrique du $\mathrm{SiO}_{2} ; \max C$ : grandeur maximale mesurée de la capacité du système électrolyte-semiconducteur. En plus, pour ce qui concerne la quantité d'électricité passant à travers la frontière $\mathrm{n}-\mathrm{Si} / \mathrm{HF}$ en polarisation anodique (où l'oxydation du Si peut être réalisée) nous mesurons qu'elle ne peut pas transformer plus de 0,05 d'une couche monoatomique de $\mathrm{Si}(j<1 \times$ $10^{-6} \mathrm{~A} . \mathrm{cm}^{-2}$ ) en supposant un taux de transformation $\mathrm{Si} \rightarrow \mathrm{SiO}_{2}$ de $100 \%$.

Quand on remplace la solution $\mathrm{HF}$ par $\mathrm{Na}_{2} \mathrm{SO}_{4}$, ou $\mathrm{H}_{2} \mathrm{SO}_{4}$, ou $\mathrm{NaOH}$ on observe une variation

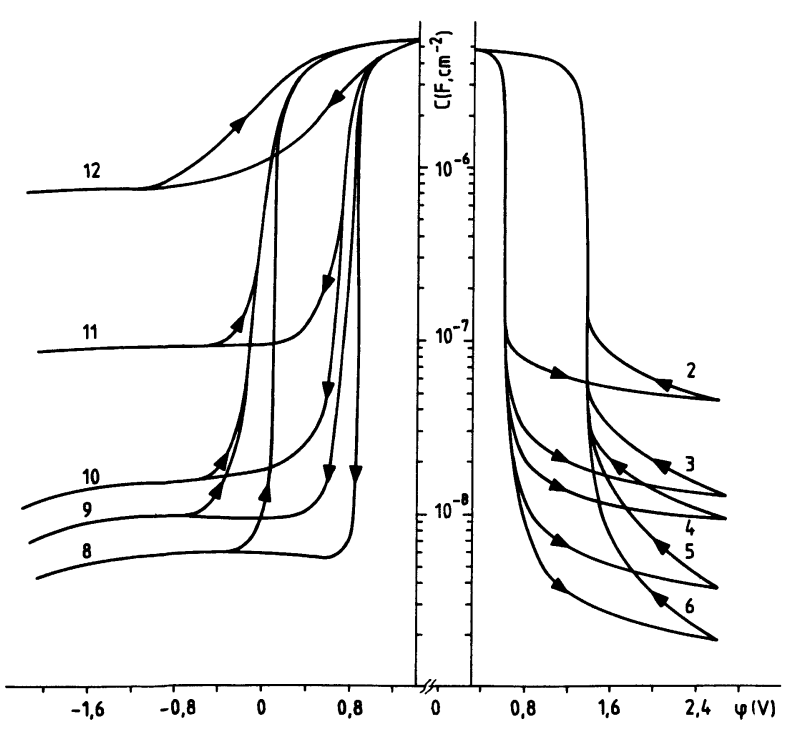

Fig. 5. - Dépendances $C(\phi)$ pour le système " surface (111) de $\mathrm{Si} / 0,1 \mathrm{~N} \mathrm{Na}_{2} \mathrm{SO}_{4}$ ". Les notations sont les mêmes que sur la figure 1 .

$\left[C(\phi)\right.$ dependence for a «(111) $\mathrm{Si}$ surface $/ 0.1 \mathrm{~N} \mathrm{Na}_{2} \mathrm{SO}_{4}$ " system. Same notations as in figure 1.] des caractéristiques $C(\phi)$ et $j(\phi)$ (Figs. 5 à 7) qui démontre la transformation de l'interface du type $\mathrm{Si}-\mathrm{F}$ en interface du type $\mathrm{Si}-\mathrm{O}-\mathrm{Si}$...-Si-OH par une oxydation anodique bien contrôlée. Sur les caractéristiques $C(\phi)$ cette transformation se traduit d'abord par la saturation des dépendances $C(\phi)$ en accumulation au niveau de $\max C=3$ à $5 \times 10^{-6} \mathrm{~F} . \mathrm{cm}^{-2}$. Ensuite les dépendances $C(\phi)$ font apparaître une hystérésis évidente. Ces deux faits résultent de la formation d'une couche mince d'oxyde, dont l'épais-. seur peut être déterminée selon la relation :

$$
d_{\mathrm{ox}}=\frac{\varepsilon_{0} \varepsilon_{\mathrm{ox}}}{\max C}=7 \text { à } 12 \AA .
$$

L'interprétation quantitative des dépendances $j(\phi)$ intermédiaires (Fig. 6b) selon [9] révèle que la trans-

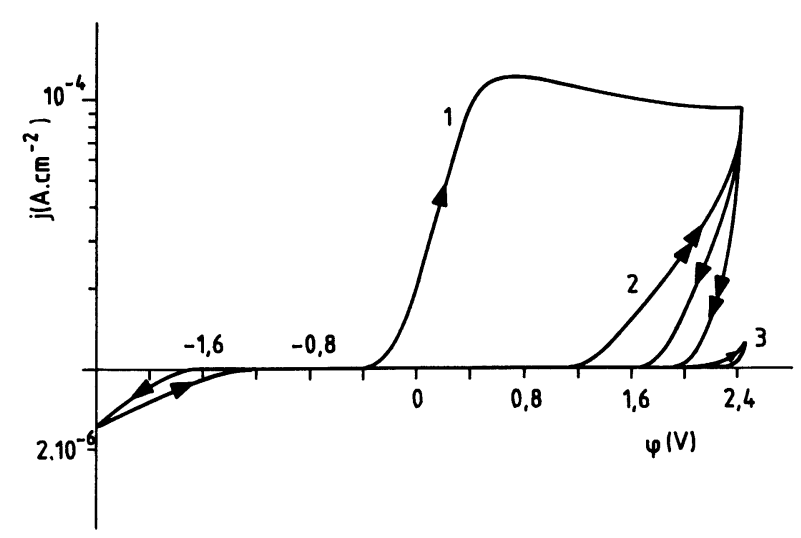

Fig. 6. - Evolution des dépendances $j(\phi)$ pour le système " surface (111) de n-Si $(10 \Omega \mathrm{cm}) / 0,1 \mathrm{~N} \mathrm{Na}{ }_{2} \mathrm{SO}_{4}$ ". 1, 2, 3 : numéros des cycles de balayage du potentiel électrochimique.

$[j(\phi)$ evolution for the system : (111) surface of n-Si $(10 \Omega \mathrm{cm}) / 0.1 \mathrm{~N} \mathrm{Na}_{2} \mathrm{SO}_{4} .1,2,3=$ number of the electrochemical potential cycle]. 


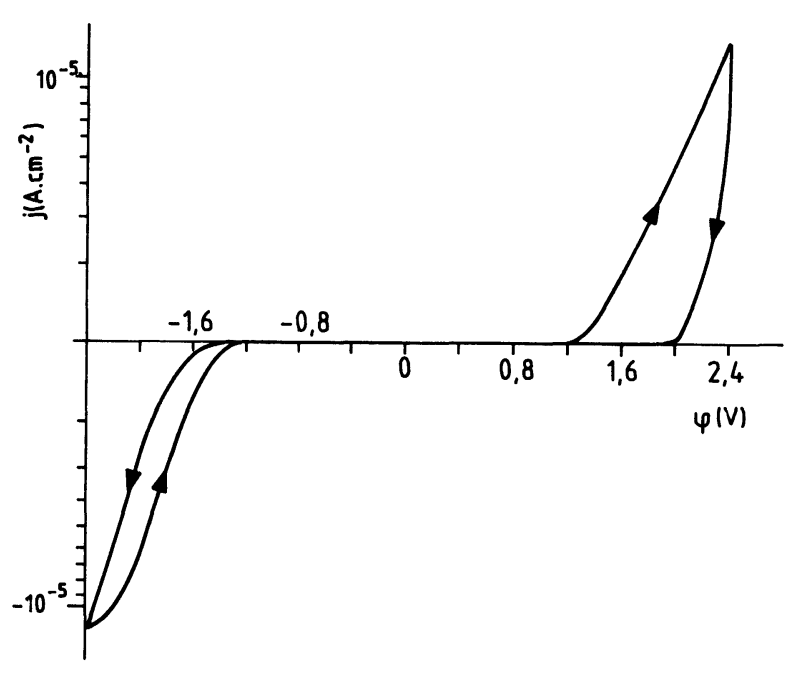

Fig. 7. - Dépendance $j(\phi)$ stabilisée pour le système : surface (111) de $n-\mathrm{Si}(10 \Omega \mathrm{cm}) / 0,1 \mathrm{~N} \mathrm{Na}_{2} \mathrm{SO}_{4}$.

$[j(\phi)$ dependence (after stabilization for the system : (111) surface of $\mathrm{n}-\mathrm{Si}(10 \Omega \mathrm{cm}) / 0.1 \mathrm{~N} \mathrm{Na}_{2} \mathrm{SO}_{4}$.]

formation électrochimique du type $\mathrm{Si}-\mathrm{F} \Rightarrow \mathrm{Si}-\mathrm{O}-\mathrm{Si}$... -Si-OH nécessite une charge électrique $Q_{1}=1,7 \times$ $10^{16} \mathrm{~cm}^{-2}$ charges élémentaires par $\mathrm{cm}^{2}$ indépendamment du type du Si. Cette charge $Q_{1}$ dépend exclusivement de l'amplitude du potentiel anodique dans son domaine initial de variation. Dans le cas présenté (Fig. $6, \max \phi=2,4 \mathrm{~V}$ ) cette charge correspond à une épaisseur d'oxyde propre $d_{\mathrm{ox}} \simeq 16 \AA$ en supposant un taux de sortie des ions $\mathrm{Si}^{4+}$ de $100 \%$. La formation de l'oxyde propre se termine presque totalement pendant le premier cycle de variation de potentiel (cathode $\rightleftarrows$ anode, Fig. 6). Après le deuxième cycle les courbes $j(\phi)$ deviennent stables et le flux électrique diminue d'un ordre de grandeur (Fig. 7). La quantité d'électricité déduite de $j(\phi)$

$$
\left(Q_{2}=\int_{t_{1}}^{t_{2}} j(\phi) \mathrm{d} t\right)
$$

est maintenant $Q_{2}=1 \times 10^{15}$ électrons $\mathrm{cm}^{-2}$. Elle est dépensée au cours de l'évolution de type : $\mathrm{Si}-\mathrm{O}-\mathrm{Si}$... -Si-OH $\Rightarrow \mathrm{Si}-\mathrm{O}-\mathrm{Si}$... $\mathrm{Si}-\mathrm{H}$ analogue à celle du germanium $[7,10,11]$. Il s'ensuit qu'on peut faire varier une épaisseur d'oxyde propre très mince sur le $\mathrm{Si}$ (dans la gamme $1 \AA$ à quelques dizaines $\AA$ ) d'une façon parfaitement contrôlable dans un système électrolyte-semiconducteur (ou EOPS).

Le germanium représente un autre type de semiconducteur avec une bande interdite assez étroite $\left(E_{\mathrm{g}}(\mathrm{Ge}) \simeq 0,7 \mathrm{eV}\right)$ dont les paramètres électrophysiques du système électrolyte-semiconducteur $\left[C, \sigma_{\square}\right.$, $\bar{\mu}_{\text {SM }}$ ] évoluent avec $\phi$ à température ambiante (298 K) pour une amplitude donnée de $\phi$, dans des conditions d'équilibre thermodynamique des trous et des électrons avec le réseau cristallin $[5,7,11]$. Les résultats expérimentaux de la figure 8 montrent la variation

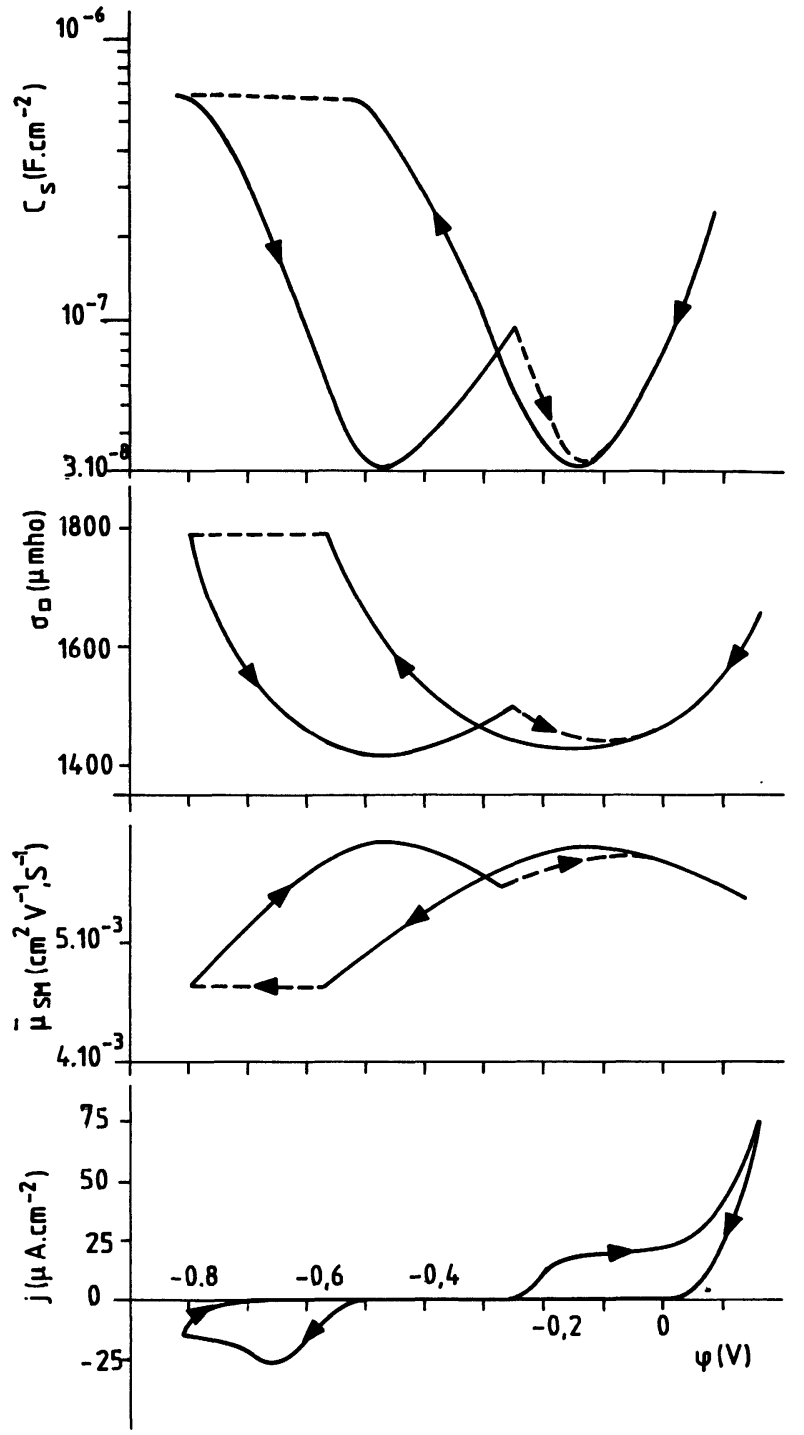

Fig. 8. - Dépendances $C(\phi), \sigma_{\square}(\phi), \bar{\mu}_{\text {SM }}(\phi)$ et $j(\phi)$ pour une électrode de $\mathrm{n}-\mathrm{Ge}(40 \Omega \mathrm{cm})$, surface (111) dans $0,1 \mathrm{~N}$ $\mathrm{Na}_{2} \mathrm{SO}_{4}$.

$\left[C(\phi), \sigma_{\square}(\phi), \bar{\mu}_{\mathrm{SM}}(\phi)\right.$ and $j(\phi)$ dependences for a n-Ge $(40 \Omega \mathrm{cm})$ electrode, (111) surface, in $0.1 \mathrm{~N} \mathrm{Na}_{2} \mathrm{SO}_{4}$.]

cyclique des caractéristiques $C(\phi), \sigma_{\square}(\phi), \bar{\mu}_{\mathrm{SM}}(\phi)$ (mobilité moyenne magnétorésistive [7]) et $j(\phi)$ qui révèlent deux structures chimiques de la surface du germanium : en polarisation anodique : Ge-O-Ge-... $\mathrm{Ge}-\mathrm{OH}$, et en polarisation cathodique : Ge-O-Ge...$\mathrm{Ge}-\mathrm{H}[7,10,11]$. On peut souligner la bonne qualité électrophysique de la surface du germanium dans ces conditions : la densité d'états rapides de surface au voisinage du milieu de $E_{\mathrm{g}}$ ne dépasse pas $N_{\mathrm{ss}}<1 \times$ $10^{10} \mathrm{~cm}^{-2}$, et la vitesse de recombination superficielle $s$ est inférieure à $20 \mathrm{~cm} \cdot \mathrm{s}^{-1}$.

Le résultat le plus important des mesures $C(\phi)$ sur des électrodes de $\mathrm{Si}$ et de $\mathrm{Ge}$ est donné par la comparaison des grandeurs expérimentales de la capacité de surface des semiconducteurs $C_{\mathrm{sc}}^{0}$ à l'équilibre $(j=0)$ dont la valeur à son tour détermine 
uniquement le potentiel de surface $V_{\mathrm{s}}^{0}$ et la charge superficielle de la zone de charge d'espace du semiconducteur $Q_{\mathrm{sc}}^{0}$ selon la théorie de surface des semiconducteurs [17, 18]. Le tableau I montre que l'absence d'oxyde propre (solution de HF) sur la surface du silicium correspond à une densité minimale de la charge superficielle de la zone de charge d'espace $\left(Q_{\mathrm{sc}} \simeq 0\right)$. Mais au contraire, cette charge augmente quand on observe (selon les variations des caractéristiques $C(\phi)$ et $j(\phi))$ la formation d'une couche mince d'oxyde propre sur le Si : $Q_{\mathrm{sc}}^{0}=-(0,4$ à 1,4$) \times 10^{12}$ électrons $\mathrm{cm}^{-2}$.

Tableau I. - Paramètres électrophysiques de la surface (111) de $\mathrm{n}-\mathrm{Si}(10 \Omega \mathrm{cm})$ dans différents électrolytes et à $j=0$.

[Electrophysical properties of the (111) surface of $\mathrm{n}$-Si $(10 \Omega \mathrm{cm})$ in different electrolytes, for $j=0$.]

\begin{tabular}{|c|c|c|c|}
\hline \multirow{2}{*}{$\begin{array}{l}\text { Solutions } \\
\text { aqueuses }\end{array}$} & \multicolumn{3}{|c|}{ Paramètres } \\
\hline & $C_{s c}^{0}\left(F \cdot \mathrm{cm}^{-2}\right)$ & $V_{s}^{0}(V)$ & $a_{s c}^{0}\left(\right.$ el.. $\mathrm{cm}^{-2}$ ) \\
\hline $\mathrm{H}_{2} \mathrm{SO}_{4} ; \mathrm{pH}=1,5$ & (1 à 5). $10^{-6}$ & 0,18 à 0,25 & $-(0,4$ à 1,4$) \cdot 10^{12}$ \\
\hline $\mathrm{Na}_{2} \mathrm{SO}_{4} ; \mathrm{pH}=6$ & (3 à 5$) .10^{-6}$ & 0,22 à 0,25 & $-(1$ à 1,4$) \cdot 10^{12}$ \\
\hline $\mathrm{NaOH} ; \mathrm{pH}=12,5$ & (3 à 5). $10^{-6}$ & 0,22 à 0,25 & $-(1$ à 1,4$) \cdot 10^{12}$ \\
\hline $\mathrm{HF}, \mathrm{pH}=1$ & $(4$ à 8$) \cdot 10^{-8}$ & $-0,01$ à 0,04 & $(-0,5$ à 2$) \cdot 10^{10}$ \\
\hline
\end{tabular}

Des résultats semblables ont été trouvés pour le germanium [7, 9]. Il s'ensuit deux conclusions importantes. Premièrement, la grandeur algébrique de la charge $Q_{\mathrm{sc}}^{0}$ n'est pas liée à l'adsorption spécifique de cations $\mathrm{H}^{+}$et $\mathrm{Na}^{+}$sur la surface de $\mathrm{Si}$ et $\mathrm{Ge}$, puisqu' une variation importante de leurs concentrations ne change pas visiblement $Q_{\mathrm{sc}}^{0}$. En même temps, dans une solution de HF où l'oxyde propre n'existe plus sur la surface de $\mathrm{Si}$, la charge $Q_{\mathrm{sc}}^{0}$ est diminuée de deux ordres de grandeur. Deuxièmement, il résulte que l'apparition d'une charge négative importante dans la zone de charge d'espace sur la surface de Si (Tableau I) et de Ge $[7,9]$ est liée directement à la formation des premières (1 à 3$)$ couches monomoléculaires d'oxyde qui portent la charge électrique :

$$
Q_{\mathrm{ox}} \simeq-Q_{\mathrm{sc}},
$$

en négligeant l'interaction entre les ions de l'électrolyte et l'oxyde propre qui présente une bande interdite assez large $\left(E_{\mathrm{g}}\left(\mathrm{SiO}_{2}\right) \simeq 9\right.$ à $10 \mathrm{eV} ; E_{\mathrm{g}}\left(\mathrm{GeO}_{2}\right) \simeq 5$ à $6 \mathrm{eV})$.

Les résultats de nos recherches sur le groupe $\mathrm{A}^{4}(\mathrm{Si}$, $\mathrm{Ge})$ montrent que la grandeur de $Q_{\mathrm{sc}}^{0}$ est presque indépendante du type de conduction et de la concentration d'impuretés mais en même temps qu'elle dépend visiblement de l'orientation cristallographique de la surface étudiée (Tableau II) et plus encore de l'adsorption d'impuretés métalliques $(\mathrm{Cu}, \mathrm{Ag}, \mathrm{Au}$, $\mathrm{Pt}, \mathrm{Pd}, \mathrm{Ni}, \mathrm{Fe}, . .$.$) sur la surface de \mathrm{Si}$ et $\mathrm{Ge}$ [12-14],
Tableau II. - Variation de la charge $Q_{\mathrm{sc}}^{0}$ selon l'orientation de la surface de $\mathrm{Si}$ et de $\mathrm{Ge}$ dans une solution $0,1 \mathrm{~N} \mathrm{Na}_{2} \mathrm{SO}_{4}$.

$\left[Q_{\mathrm{sc}}^{0}\right.$ charge variation with the surface orientation of $\mathrm{Si}$ and $\mathrm{Ge}$ in a $0.1 \mathrm{~N} \mathrm{Na}_{2} \mathrm{SO}_{4}$ solution.]

\begin{tabular}{|l|l|l|}
\hline \multicolumn{2}{|l|}{$\begin{array}{l}\text { Surface du } \\
\text { semiconducteur }\end{array}$} & $\begin{array}{c}Q_{\text {sc }}^{0} \\
\text { charges élém. cm-2 }\end{array}$ \\
\hline \multirow{2}{*}{$\mathrm{Si}$} & $(100)$ & $-0,3 \times 10^{12}$ \\
\cline { 2 - 3 } & $(111)$ & $-1 \times 10^{12}$ \\
\hline \multirow{2}{*}{ Ge } & $(100)$ & $-3 \times 10^{10}$ \\
\cline { 2 - 3 } & $(111)$ & $-6 \times 10^{10}$ \\
\hline
\end{tabular}

qui augmentent la densité d'états de surface rapides, profonds et discrets. Comme l'ont montré nos études [12 à 16], tous les métaux adsorbés (ou dégagés) sur la surface propre des différents semiconducteurs sous forme d'atomes neutres $\left(\mathrm{Me}_{\mathrm{sol}}^{\mathrm{n}+}+\mathrm{ne}^{-} \rightarrow \mathrm{Me}_{\mathrm{ads}}^{0}\right)$ fournissent un spectre d'états de surface qui interviennent dans les processus de capture, de recombinaison et de collision.

\section{Résultats sur les semiconducteurs binaires $A^{3} B^{5}(\operatorname{In} P$, InAs, InSb ; GaN, GaP, GaAs, GaSb).}

Pour tous les semiconducteurs $A^{3} B^{5}$ dans des solutions aqueuses, de même que pour les semiconducteurs $\mathrm{A}^{4}$ on observe des zones de potentiel $\phi$ où les variations de $C, \sigma_{\square}, j$ restent réversibles et stables indépendamment du nombre de balayage du potentiel. D'après les courbes expérimentales $C(\phi)$ et $\sigma_{\square}(\phi)$, les matériaux du groupe $A^{3} B^{5}$ peuvent être divisés en deux ensembles. D'abord ceux analogues du Ge, représentés dans nos études par InAs, InSb, GaSb (Figs. 9 à 12) : ils possèdent une bande interdite relativement étroite $\left(E_{\mathrm{g}} \leqslant 1 \mathrm{eV}\right)$, et les mesures électrophysiques sur le système EOPS peuvent être effectuées dans des conditions d'équilibre thermodynamique entre le gaz électron-trou et le réseau cristallin sur un domaine de variation du potentiel électrochimique bien déterminé.

Ensuite les semiconducteurs, analogues du silicium, comme InP, GaN, GaP, GaAs (Figs. 13 et 14), qui ont une bande interdite large $\left(E_{\mathrm{g}} \geqslant 1 \mathrm{eV}\right)$ : les mesures électrophysiques sur le système EOPS s'y déroulent toujours dans des conditions de déséquilibre par désertion des porteurs minoritaires de charge électrique. On peut éviter cette désertion et atteindre l'inversion pour ces matériaux en les éclairant fortement par une lumière susceptible d'être absorbée (Fig. 13).

La variation des courbes $C(\phi)$ (Figs. 9, 10) en fonction du $\mathrm{pH}$ des solutions nous montre qu'il existe 


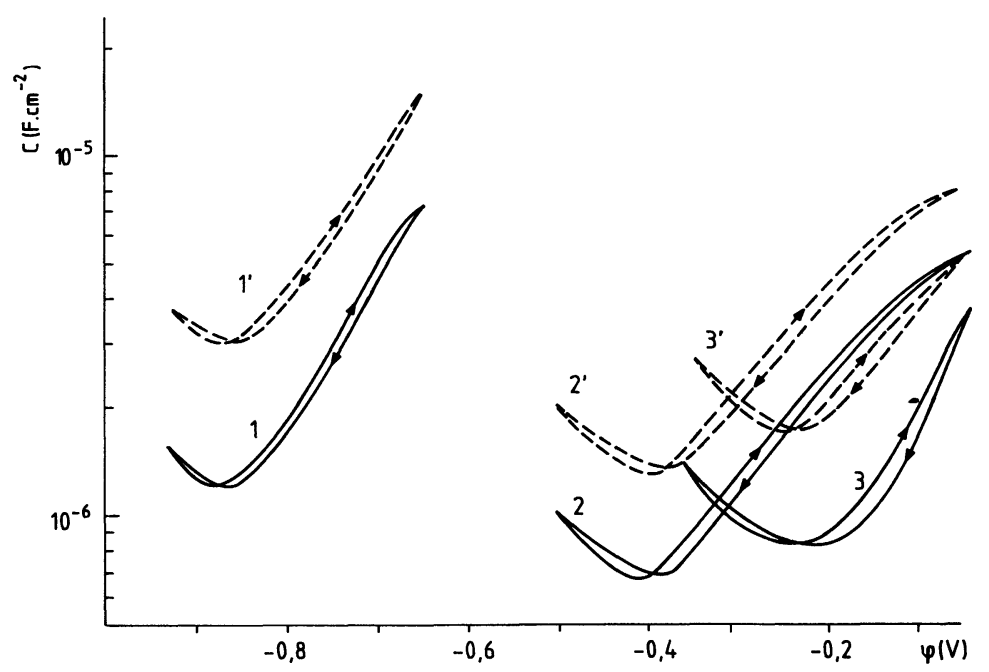

Fig. 9. - Dépendances $C(\phi)$ pour une électrode de p-InSb $\left(2 \times 10^{12} \mathrm{~cm}^{-3}\right)$; orientation de la surface : plan $(211)-\mathrm{A}^{3}:$ $1^{\prime}, 2^{\prime}, 3^{\prime}$ et plan $(\overline{211})-\mathrm{B}^{5}: 1,2,3$; dans $0,1 \mathrm{~N} \mathrm{NaOH}, \mathrm{pH}=11: 1,1^{\prime} ;$ dans $0,1 \mathrm{~N} \mathrm{Na}_{2} \mathrm{SO}_{4}, \mathrm{pH}^{\prime}=5,5: 2,2^{\prime}$ et dans $0,1 \mathrm{~N} \mathrm{H}_{2} \mathrm{SO}_{4}, \mathrm{pH}=1,5: 3,3^{\prime}$.

$\left[C(\phi)\right.$ dependence for a p-InSb $\left(2 \times 10^{12} \mathrm{~cm}^{-3}\right)$ electrode ; surface orientation : $\mathrm{A}^{3}(211)$ plane : $1^{\prime}, 2^{\prime}, 3^{\prime}$ and $\mathrm{B}^{5}(\overline{211})$ plane : $1,2,3$; in $0.1 \mathrm{~N} \mathrm{NaOH}, \mathrm{pH}=11 ; 1,1^{\prime} ; 0.1 \mathrm{~N} \mathrm{Na}_{2} \mathrm{SO}_{4}, \mathrm{pH}=5.5: 2,2^{\prime} ; 0.1 \mathrm{~N} \mathrm{H}_{2} \mathrm{SO}_{4}, \mathrm{pH}=1.5: 3,3^{\prime}$.]

une chute de potentiel dans la couche de Helmholtz, créée par la dissociation des complexes superficiels du type $\mathrm{Si}_{\text {surf }}-\mathrm{OH} \rightleftarrows \mathrm{Si}-\mathrm{O}^{-}+\mathrm{H}^{+}$. Dans des conditions idéales déterminées par l'équation de Nernst ce processus donne : $\frac{\Delta \phi}{\Delta \mathrm{pH}}=60 \mathrm{mV} / \mathrm{pH}$ à $300 \mathrm{~K}$. Nous

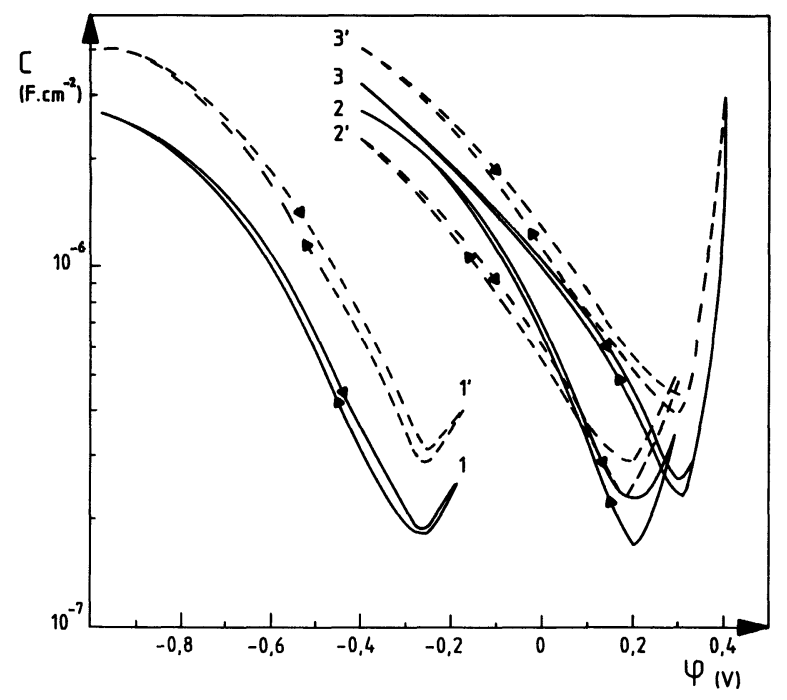

Fig. 10. - Dépendance $C(\phi)$ pour une électrode de nInAs $\left(2 \times 10^{16} \mathrm{~cm}^{-3}\right)$; orientation de la surface : plan (111)-A $\mathrm{A}^{3}: 1,2,3$ et plan (111)-B $\mathrm{B}^{5}: 1^{\prime}, 2^{\prime}, 3^{\prime}$; dans 0 , $1 \mathrm{~N} \mathrm{NaOH}, \mathrm{pH}=11: 1,1^{\prime}$; dans $0,1 \mathrm{~N} \mathrm{Na}_{2} \mathrm{SO}_{4}, \mathrm{pH}=5,5$ : $2,2^{\prime}$ et dans $0,1 \mathrm{~N} \mathrm{H}_{2} \mathrm{SO}_{4}, \mathrm{pH}=1,5: 3,3^{\prime}$.

$\left[C(\phi)\right.$ dependence for a $\mathrm{n}$-InAs $\left(2 \times 10^{16} \mathrm{~cm}^{-3}\right)$ electrode; surface orientation : $A^{3}$ (111) plane : 1, 2, 3 and $B^{5}(111)$ plane : $1^{\prime}, 2^{\prime}, 3^{\prime}$; in $0.1 \mathrm{~N} \mathrm{NaOH}, \mathrm{pH}=11: 1,1^{\prime}$; $0.1 \mathrm{~N} \mathrm{Na}_{2} \mathrm{SO}_{4}, \mathrm{pH}=5.5: 2,2^{\prime} ; 0.1 \mathrm{~N} \mathrm{H}_{2} \mathrm{SO}_{4}, \mathrm{pH}=1,5$ : $3,3^{\prime}$.] avons obtenu pour le sous-groupe « In » des composés $\mathrm{A}^{3} \mathrm{~B}^{5}: \frac{\Delta \phi}{\Delta \mathrm{pH}}(\mathrm{InSB})=65 \mathrm{mV} / \mathrm{pH}, \frac{\Delta \phi}{\Delta \mathrm{pH}}(\mathrm{InAs})=$ $60 \mathrm{mV} / \mathrm{pH}, \frac{\Delta \phi}{\Delta \mathrm{pH}}(\mathrm{InP})=35 \mathrm{mV} / \mathrm{pH}$, ce qui montre que le phénomène dépend de la nature chimique du semiconducteur.

En comparant les résultats des calculs (résolution de l'équation de Poisson) et les valeurs expérimentales

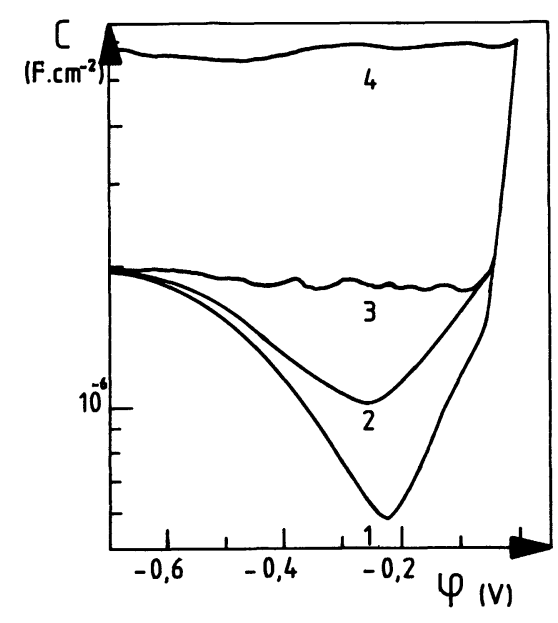

Fig. 11. - Dépendances $C(\phi)$ pour une électrode de nInSb $\left(1 \times 10^{14} \mathrm{~cm}^{-3}\right)$; orientation de la surface : plan (211)- $\mathrm{B}^{5}$; dans une solution $0,05 \mathrm{~N} \mathrm{HBF}_{4}$ pure (1), avec $10^{-8} \mathrm{M} \mathrm{Cu}(2)$; avec $10^{-7} \mathrm{M} \mathrm{Cu}(3)$ et avec $10^{-6} \mathrm{M} \mathrm{Cu}$ (4).

$\left[C(\phi)\right.$ dependence for a $\mathrm{n}$-InSb $\left(1 \times 10^{14} \mathrm{~cm}^{-3}\right)$ electrode; surface orientation : $\mathrm{B}^{5}(\overline{211})$ plane; in $0.05 \mathrm{~N} \mathrm{HBF}_{4}$ pure (1), with $10^{-8} \mathrm{M} \mathrm{Cu}$ (2), $10^{-7} \mathrm{M} \mathrm{Cu}$ (3) or $10^{-6} \mathrm{M} \mathrm{Cu}$ (4).] 


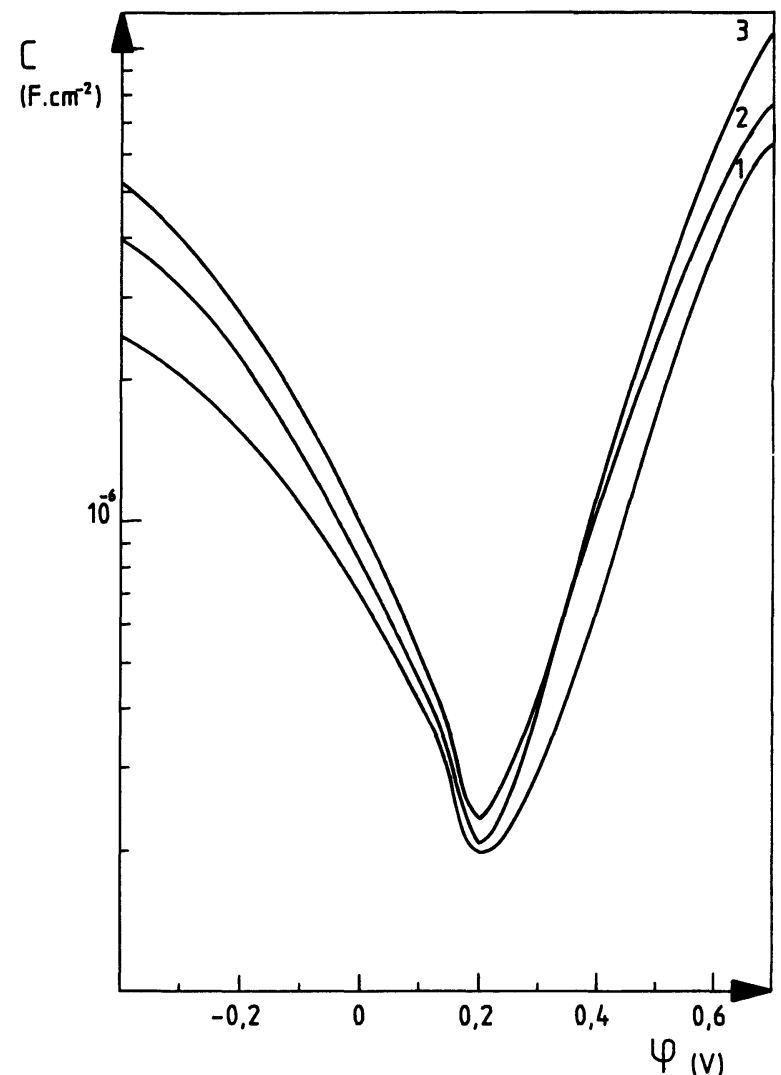

Fig. 12. - Dépendances $C(\phi)$ pour une électrode de nInAs $\left(2 \times 10^{16} \mathrm{~cm}^{-3}\right)$; orientation de la surface : plan (111)- $\mathrm{A}^{3}$; dans une solution $0,1 \mathrm{~N} \mathrm{Na}_{2} \mathrm{SO}_{4}$ pure (1), avec $10^{-8} \mathrm{M} \mathrm{Cu}(2)$ et avec $10^{-7} \mathrm{M} \mathrm{Cu}(3)$.

$\left[C(\phi)\right.$ dependence for a n-InAs $\left(2 \times 10^{16} \mathrm{~cm}^{-3}\right)$ electrode; surface orientation : $A^{3}$ (111) plane; in $0.1 \mathrm{~N} \mathrm{Na}_{2} \mathrm{SO}_{4}$ pure (1), with $10^{-8} \mathrm{M} \mathrm{Cu}$ (2) or $10^{-7} \mathrm{M} \mathrm{Cu}$ (3).]

de la capacité au minimum des fonctions $C(\phi)$ nous avons trouvé (Figs. 9, 10), que pour les solutions neutres $(\mathrm{pH}=5,5)$ et pour les surfaces de InSb et InAs bien polies on a :

$$
\min C^{\exp } \simeq \min C_{\mathrm{sc}}^{\text {theor. }} .
$$

Cela nous permet d'évaluer la densité d'états de surfaces rapides au voisinage du milieu de la bande interdite :

$$
\begin{gathered}
N_{\text {ssmin }}(\operatorname{InSb})<10^{11} \mathrm{~cm}^{-2}, \\
N_{\text {ssmin }}(\operatorname{InAs})<5 \times 10^{10} \mathrm{~cm}^{-2} .
\end{gathered}
$$

Il s'ensuit qu'on obtient une interface OPS de bonne qualité en la formant en régime potentiodynamique par oxydation anodique, quand $d_{\mathrm{ox}}<50 \AA[15,16]$. Il est évident d'après les figures 9,10 qu'une variation de $\mathrm{pH}$ de la solution modifie la grandeur $N_{\text {ssmin }}$ mais de manière différente pour InSb et InAs. Cela veut dire que la densité d'états rapides de surface $N_{\text {ssmin }}$

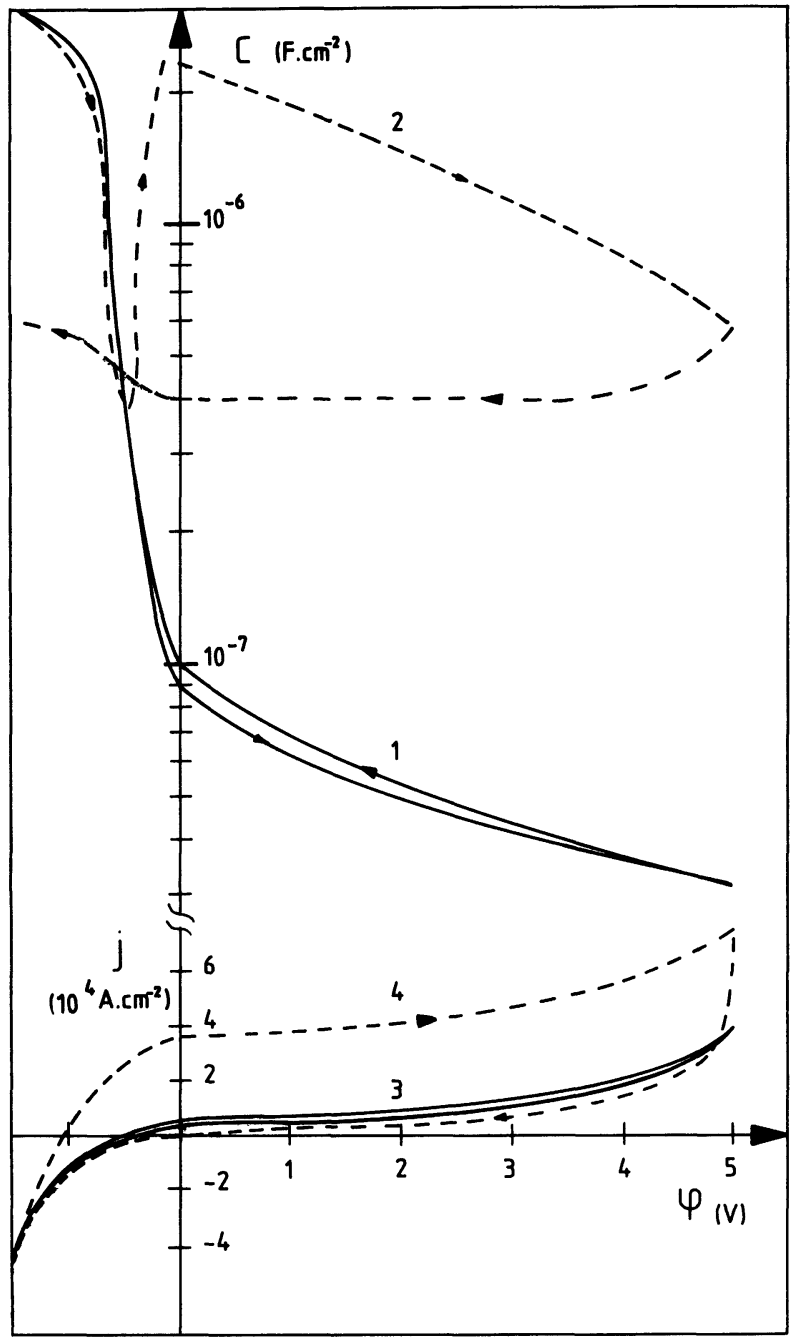

Fig. 13. - Dépendances $C(\phi): 1,2$ et $j(\phi): 3,4$ pour une électrode de $\mathrm{n}$-InP $\left(4,5 \times 10^{16} \mathrm{~cm}^{-3}\right)$; orientation de la surface : plan (111)- $\mathrm{B}^{5}$ dans une solution $0,1 \mathrm{~N} \mathrm{NaOH}$ en obscurité $(1,3)$ et sous éclairement $(2,4)$.

$[C(\phi): 1,2$ and $j(\phi): 3,4$ dependences for a $n-\operatorname{InP}(4.5 \times$ $10^{16} \mathrm{~cm}^{-3}$ ) electrode; surface orientation : $\mathbf{B}^{5}(\overline{111})$ plane in $0.1 \mathrm{~N} \mathrm{NaOH}$; in darkness $(1,3)$ or under illumination $(2,4)$.]

sur l'interface OPS dépend en même temps de la nature chimique du semiconducteur et du $\mathrm{pH}$ de l'électrolyte.

On peut augmenter la densité d'états rapides de l'interface OPS de façon beaucoup plus importante par adsorption de divers métaux $(\mathrm{Cu}, \mathrm{Au}, \mathrm{Pt}$...) introduits dans l'électrolyte (Figs. 11, 12); l'augmentation de $N_{\mathrm{ss}}$ dépend directement de la quantité d'impuretés introduites dans l'électrolyte. Les résultats nous montrent (Figs. 11, 12) que l'adsorption d'un même métal crée des états d'interface ayant différentes positions dans la bande interdite pour InSb et InAs. L'adsorption de petites quantités de métaux provoque en général le même phénomène à l'interface OPS pour les semi- 


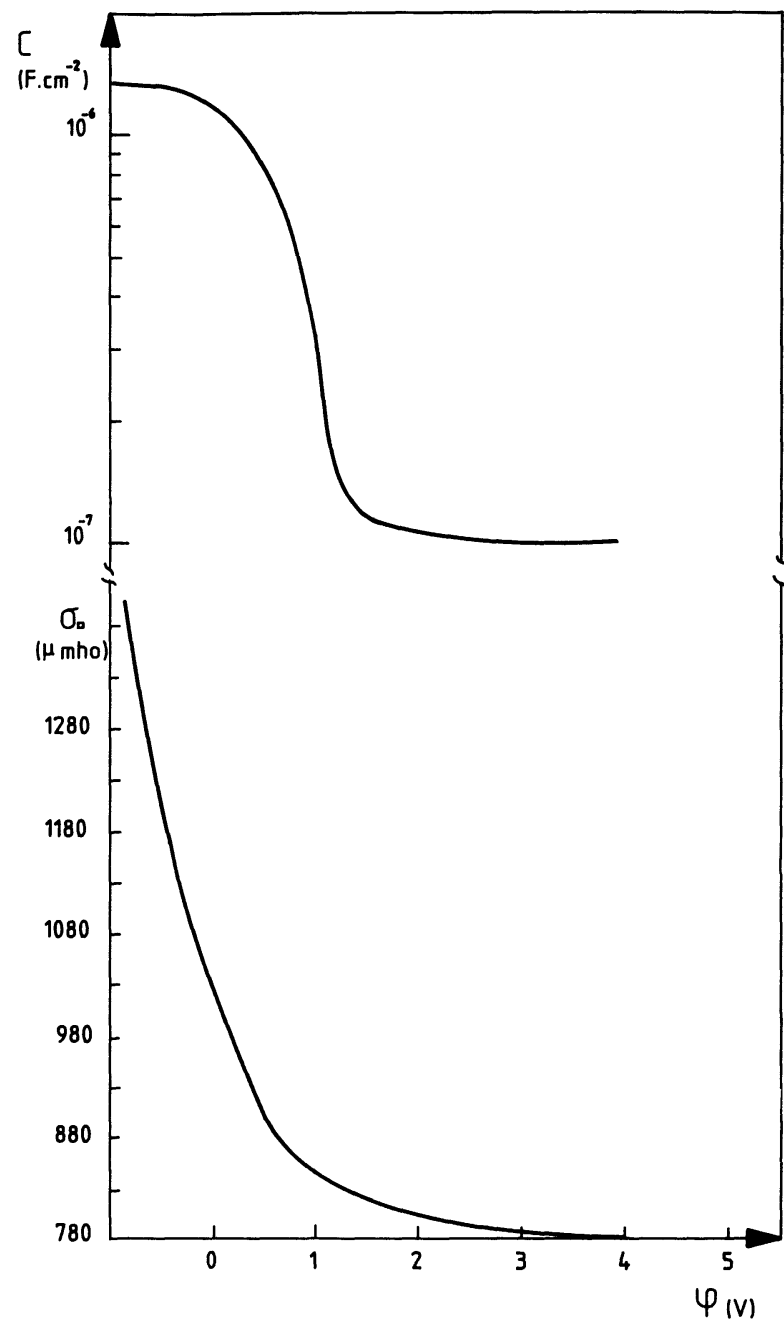

Fig. 14. - Dépendances $C(\phi)$ et $\sigma_{\square}(\phi)$ pour une électrode n-GaN $\left(\sim 10^{18} \mathrm{~cm}^{-3}\right)$ dans une solution $0,1 \mathrm{~N} \mathrm{Na}_{2} \mathrm{SO}_{4}$.

$\left[C(\phi)\right.$ and $\sigma_{\square}(\phi)$ dependences for a n-GaN $\left(\sim 10^{16} \mathrm{~cm}^{-3}\right)$ electrode in $0.1 \mathrm{~N} \mathrm{Na}_{2} \mathrm{SO}_{4}$.]

conducteurs du groupe $A^{3} B^{5}$, et pour les matériaux analogues du groupe $\mathrm{A}^{4}$.

En comparant les grandeurs de $\left.Q_{\mathrm{sc}}^{0}\right|_{j=0}$, déterminées par les mesures de $\left.C(\phi)\right|_{j=0}$, et en supposant que $Q_{\mathrm{sc}}^{0}=-Q_{\mathrm{ox}}$ pour un oxyde propre d'épaisseur $d_{\mathrm{ox}}<50 \AA$, on peut constater la régularité de la variation de $Q_{\mathrm{ox}}$ avec les propriétés cristallo-physicochimiques des semiconducteurs des groupes $\mathrm{A}^{4}$ et $\mathrm{A}^{3} \mathrm{~B}^{5}$.

\section{Résumé des résultats expérimentaux.}

4.1. - La zone de localisation de la charge $Q_{\text {ox }}$ au voisinage de l'interface OPS est 7 à $16 \AA$ pour le Si et moins de $50 \AA$ pour les autres semiconducteurs étudiés recouverts par un oxyde propre mince anodique.
4.2. - En l'absence d'impuretés métalliques, la nature de cette charge $Q_{\mathrm{ox}}$ est "génétique " (intrinsèque) et est vraisemblablement déterminée par le mécanisme de formation de l'oxyde propre sur la surface des semiconducteurs, qui résulte de la correspondance cristallo-physico-chimique des réseaux du semiconducteur et de l'oxyde propre.

4.3. - Pour les semiconducteurs du groupe $\mathrm{A}^{4}$ ayant une même orientation cristallographique de la surface on observe que :

$$
Q_{\mathrm{ox}}(\mathrm{Si})>Q_{\mathrm{ox}}(\mathrm{Ge})>0 \text {. }
$$

4.4. - La grandeur $Q_{o x}$ dépend faiblement du type et du dopage des matériaux $\mathrm{A}^{4}$ mais elle varie clairement avec l'orientation cristallographique de la surface étudiée :

$$
\begin{aligned}
& Q_{\mathrm{ox}}^{(111)}(\mathrm{Si})>Q_{\mathrm{ox}}^{(100)}(\mathrm{Si})>0 \\
& Q_{\mathrm{ox}}^{(111)}(\mathrm{Ge})>Q_{\mathrm{ox}}^{(100)}(\mathrm{Ge})>0
\end{aligned}
$$

4.5. - La grandeur et le signe de $Q_{\mathrm{ox}}$, de même que la distribution des états de surface dans la bande interdite des semiconducteurs $A^{4}$ et $A^{3} B^{5}$ dépendent fortement de l'adsorption sur leurs surfaces des métaux lourds : $\mathrm{Cu}, \mathrm{Ag}, \mathrm{Au}, \mathrm{Fe}, \mathrm{Co}, \mathrm{Ni}, \mathrm{Pt}, \mathrm{Pd}, \ldots$

4.6. - Dans des conditions identiques de formation de l'oxyde propre, on observe une variation régulière de $Q_{\text {ox }}$ pour les semiconducteurs du sous-groupe « In » de $A^{3} B^{5}$, avec l'élément $B^{5}$ :

$$
Q_{\mathrm{ox}}(\operatorname{InP})>Q_{\mathrm{ox}}(\mathrm{InAs})>0>Q_{\mathrm{ox}}(\mathrm{InSb}) .
$$

4.7. - Dans des conditions identiques de formation de l'oxyde propre, on observe une variation régulière de $Q_{\text {ox }}$ pour des matériaux du sous-groupe « $\mathrm{Ga}$ » de $A^{3} B^{5}$, avec l'élément $B^{5}$ :

$Q_{\mathrm{ox}}(\mathrm{GaN})>0>Q_{\mathrm{ox}}(\mathrm{GaP})>Q_{\mathrm{ox}}(\mathrm{GaAs})>Q_{\mathrm{ox}}(\mathrm{GaSb})$.

4.8. - Pour les semiconducteurs du groupe $A^{3} B^{5}$, on observe que :

$$
Q_{\mathrm{ox}}\left(\operatorname{InB}^{5}\right)>Q_{\mathrm{ox}}\left(\mathrm{GaB}^{5}\right)
$$

quand l'élément $\mathrm{B}^{5}$ est identique.

4.9. - Pour les semiconducteurs du type $A^{3} B^{5}$ on observe l'influence de la direction de l'axe [111] sur la valeur de $Q_{\text {ox }}$ pour le même matériau

$$
Q_{\mathrm{ox}}(111) \not \equiv Q_{\mathrm{ox}}(\overline{111}) \text {. }
$$


On constate donc que les résultats obtenus pour le silicium sont déjà bien connus pour l'interface OPS préparée par oxydation thermique, ce qui nous démontre la nature commune des interfaces OPS formées par oxydation thermique ou oxydation anodique. Tous ces résultats révèlent la nature cristallophysico-chimique de l'interface OPS, dont une conception générale approchée sera donnée dans la seconde partie de notre travail.

\section{Remerciements.}

Je remercie beaucoup $M$. A. Chovet, enseignant à l'ENSERG (Institut National Polytechnique de Grenoble) pour son aide lors de la rédaction de ce texte. Je remercie d'autre part MM. G. Kamarinos, G. Pananakakis, A. Chovet, et S. Cristoloveanu (du Laboratoire de Physique des Composants à Semiconducteurs de l'ENSERG) pour les utiles discussions concernant le thème de cette publication.

\section{Bibliographie}

[1] RJanov, A. V., Edit., 7e symposium sur les processus électroniques sur les surfaces des semiconducteurs (en Russe), Acad. Sc., Novosibirsk (1980).

[2] Merz, W. J., Edit., 11th Solid State Device Research Conference (ESSDERC), European Phyșical Society, Toulouse (1981).

[3] Pantelides, S. T., Edit., The physics of $\mathrm{SiO}_{2}$ and its interfaces (Pergamon Press, N.Y.) 1978.

[4] Wilson, B., Edit., Physics of semiconductors (Acad. Press., London) 1979.

[5] Romanov, O. V., Konorov, P. P., Sov. Phys. Solid State 8 (1966) 9-14.

BohneNKAMP, K., ENGell, H. J., Ztschr. Elektrochem. 61 (1957) 1184-1196.

Brattain, W. H., BodDy, P. J., J. Electrochem. Soc. 109 (1962) 574-583.

Boddy, P. J., Brattain, W. H., J. Electrochem. Soc. 110 (1963) 570-581.

Efimov, E. A., Erusalimchik, T. G., Electrochemistry of semiconductors (Sigma Press, Washington) 1963.

[6] Romanov, O. V., Sokolov, M. A., Sultanmagomedov, S. N., Sov. Electrochem. 16 (1980) 797-804.

Memming, R., Schwandt, G., Surface Sci. 4 (1966) 109-118.

Chazalviel, J.-N., Surface sci. 88 (1979) 204-220.

Laflère, W. H., van Meirhaeghe, R. L., Cardon, F., GOMES, W. P., Surface Sci. 59 (1976) 401-409.

Medou, M. J., Cardon, F., Gomes, W. P., J. Electrochem. Soc. 124 (1977) 1623-1630.

Sculfort, J. L., Baticle, A. M., Gautron, J., C.R. Hebd. Séan. Acad. Sci. C-287 (1978) 317-322.

Etchebery, A., Etman, M., Fotouhi, B., Gautron,
J., Sculfort, J.-L., Lemasson, Ph., J. Appl. Phys. 53 (1982) 8867-8873.

[7] Romanov, O. V., UritskiY, V. J., Grusinov, B. F., Sov. Phys. Semicond. 3 (1969) 1183-1184.

[8] LaLov, J. J., Edit., Modern problems of the $\mathrm{Si} / \mathrm{SiO}_{2}$ interface (Bulg. Acad. Sci., Sofia) 1981.

[9] Romanov, O. V., Sov. Phys. Semicond. 16 (1982) 266270.

[10] Gerischer, H., Hoffman-Perez, M., Mindt, W., Bunsengess. Phys. Chem. 69 (1965) 130-139.

[11] Pleskov, Yu. V., Prog. Surface Sci. 7 (1973) 93-137.

[12] Romanov, O. V., Sokolov, M. A., Konorov, P. P., ANDREEV, A. D., Mikroelektronika (en russe) 6 (1977) 340-346.

[13] Romanov, O. V., Sokolov, M. A., Sultanmagomedov, S. N., 3e Colloque sur la Physico-Chimie des surfaces des semicond. (en russe) Acad. Sci. Novosibirsk (1978).

[14] Romanov, O. V., Sultanmagomedov, S. N., Vestnik Leningradskogo Universiteta, phys.-chem. (en russe) 2 (1980) 46-52.

[15] Romanov, O. V., Sazonov, S. G., Sokolov, M. A., Balakirev, V. V., Popov, A. V., Motaleva, N. V., Poverhnost (en russe) 2 (1983) 95-102.

[16] Romanov, O. V., Sazonov, S. G., Popov, A. V., SoKolov, M. A., Vestnik. Leningradskogo Universiteta, phys.-chem. (en russe 5 (1983) 73-78.

[17] Garrett, C. G. B., Brattain, W. H., Phys. Rev. 99 (1955) 376-396.

[18] Many, A., Goldstein, Y., Grover, N. B., Semiconductor surfaces (North-Holland Publishing Co., Amsterdam) 1965. 